\title{
Two-photon absorption induced nanowelding for assembling ZnO nanowires with enhanced photoelectrical properties
}

\author{
Cite as: Appl. Phys. Lett. 115, 103101 (2019); doi: 10.1063/1.5116242 \\ Submitted: 23 June 2019 - Accepted: 20 August 2019 • \\ Published Online: 3 September 2019
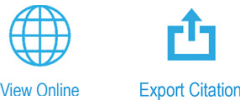 \\ Songling Xing, ${ }^{1, a)}$ (D) Luchan Lin,, ${ }^{2, a)}$ Guisheng Zou, Walter W. Duley, ${ }^{3,4}$ Lei Liu, ${ }^{1, b)}$ (D) and Y. Norman Zhou ${ }^{1,3}$

\begin{abstract}
AFFILIATIONS
'Department of Mechanical Engineering, State Key Laboratory of Tribology, Key Laboratory for Advanced Manufacturing by Materials Processing Technology, Ministry of Education of People's Republic of China, Tsinghua University, Beijing 100084, People's Republic of China

${ }^{2}$ Swiss Federal Laboratories for Materials Science and Technology (Empa), Überlandstrasse 129, 8600 Dübendorf, Switzerland

${ }^{3}$ Centre for Advanced Materials Joining, University of Waterloo, Waterloo, Ontario N2L 3G1, Canada

${ }^{4}$ Department of Physics and Astronomy, University of Waterloo, Waterloo, Ontario N2L 3G1, Canada
\end{abstract}

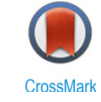

${ }^{\text {a) }}$ Contributions: S. Xing and L. Lin contributed equally to this work.

${ }^{\text {b) }}$ Author to whom correspondence should be addressed: liulei@tsinghua.edu.cn

\begin{abstract}
The joining of semiconductor nanowires (NWs) is fundamental for the construction and assembly of high performance nanoelectronic devices, but the development of reliable methods of nanojoining and nanowelding of these components has been elusive to date. In this work, we report a methodology for laser welding of wide bandgap NWs based on two-photon absorption. Two photon excitation during femtosecond laser irradiation leads to the generation of excitons forming an electron-hole plasma. As an application of this technique, we show that two-photon excitation is effective in the nanowelding of two $\mathrm{ZnO} \mathrm{NWs}$. A nanoweld, resulting in the formation of an interconnected structure, occurs when the energy in the solid state plasma is deposited in the contact area between the two ZnO NWs. During excitation with ultrashort laser pulses, rapid melting and solidification result in the generation and freezing out of oxygen vacancies in the irradiated area and the region near the contact between the two components. This enhances exciton trapping and energy deposition at the contact, facilitating the formation of a bond between the two NWs. It is also found that the absorption of visible light is significantly increased in $\mathrm{ZnO}$ NW structures assembled via two-photon femtosecond laser processing. In addition, the junction between two ZnO NWs created in this way exhibits a photoresponse that is not present prior to nanojoining. These results indicate that two-photon excitation is a promising technique for the selective deposition of thermal energy in semiconductor NWs in the absence of plasmonic interactions.
\end{abstract}

Published under license by AIP Publishing. https://doi.org/10.1063/1.5116242

Nanowelding is of great importance in the fabrication of the latest generation of complex electronic devices such as transparent conductors, ${ }^{1}$ wearable electronics, ${ }^{2}$ and memristors. ${ }^{3}$ Recent developments in nanowelding technology have been applied to joining metal-metal, ${ }^{4}$ metal-semiconductor, ${ }^{3}$ and metal-graphene ${ }^{5}$ systems. Most techniques are restricted to welding operations where at least one component is metallic, and there is little information on the welding of nanosemiconductors. As most semiconductors have high melting temperatures, joining is obtained using metallic solders and heat treatment. ${ }^{6}$ A disadvantage of using metallic solders is that they may introduce impurities which can negatively affect the overall performance of the device. Zhang et al. . $^{7}$ have reported successful nanowelding under electron beam irradiation of $\mathrm{MgO}, \mathrm{CuO}$, and $\mathrm{V}_{2} \mathrm{O}_{5}$ nanowires (NWs) without metallic solder. Welded joints were found to have a tensile strength greater than the underlying material, but welding must be carried out in a $\mathrm{CO}_{2}$ atmosphere and complex sample preparation is required prior to welding. All these previous studies of nanowelding of semiconductor materials have focused on the characteristics of the welding process itself, while limited information is available on the electrical properties of the resulting nanojunctions. ${ }^{8}$

Laser nanowelding is now recognized as an alternative to conventional methodologies for noncontact processing and high-resolution manufacturing without the need for special ambient conditions. ${ }^{9,10}$ Liu et al. ${ }^{11}$ have reported a sacrificial solder based method for nanowelding of $\mathrm{ZnO}$ NWs using Nd: YAG laser irradiation, but no details were given on the mechanism. Laser nanowelding in a metal-semiconductor 
system usually proceeds through plasmon excitation in the metallic component followed by transfer of absorbed energy to the junction, ${ }^{1}$ but this is generally impossible when welding two semiconductors. Effective joining of semiconductor components still requires a deeper understanding of the light-matter interaction during excitation with high intensity short duration laser pulses.

$\mathrm{ZnO}$ is a typical semiconductor and has been of much interest because it is readily synthesized in NW form. It is also widely used as photodetectors, transistors, and solar cells after being assembled as a random network. ${ }^{12-14}$ The photoresponse of pristine $\mathrm{ZnO}$ is limited to ultraviolet wavelengths because of its wideband gap, but its photoresponse in the visible spectral region can be extended by controlling the concentration and type of defect level. As a result, $\mathrm{ZnO}$ can be configured to be an excellent photodetector over a wide wavelength range. ${ }^{15}$ The photoresponse can be improved by semiconductor coupling, ${ }^{16}$ chemical doping, ${ }^{17}$ and the production of oxygen vacancies through the thermal decomposition of $\mathrm{ZnO}_{2}$ precursors via high temperature annealing. ${ }^{18}$ While these processes are effective, widening the wavelength range to obtain an efficient photoresponse in $\mathrm{ZnO}$ is still a challenge.

In this letter, we outline a simple method based on irradiation with a femtosecond laser for nanowelding individual $\mathrm{ZnO}$ NWs. The results show that two-photon absorption leading to the creation of a high exciton density plays a key role in this process. This is accompanied by the formation of defects, particularly in the vicinity of the contact area between two $\mathrm{ZnO} \mathrm{NWs}$. The increased concentration of these defects in laser irradiated/welded $\mathrm{ZnO} \mathrm{NW}$ structures is shown to lead to improvements in the electrical and optical properties that are important in the design of functional devices such as photodetectors for visible light.

Figure 1(a) illustrates a schematic of the experimental setup and the nanowelding procedure (see Methods in the supplementary material for all experimental details). Figures 1 (b)-1(d) show scanning electron microscopy (SEM) images collected before and after irradiation for $30 \mathrm{~s}$ at a fluence of $77.6 \mathrm{~mJ} \mathrm{~cm} \mathrm{~cm}^{-2}$. Before irradiation, individual $\mathrm{ZnO}$ NWs are clearly in physical contact, but after irradiation, the morphology changes substantially as the NWs are welded to each other. Both X- [Fig. 1(c)] and Y-[(Fig. 1(d)] shaped junctions show the presence of a filet consistent with surface melting in contact regions. Apart from the weld area, the $\mathrm{ZnO}$ NWs appear to be unaffected by irradiation, and no additional morphological changes are observed on prolonged irradiation (see Fig. S1 in the supplementary material).

Figure 2 shows transmission electron microscopy (TEM) images of a representative junction after nanowelding. The TEM lamella is lifted-out along the dashed line in the inset in Fig. 2(a), indicating partial surface melting around the contact area rather the entire NW. Both the NWs provide the "filler" material required to fill the gap between them [Fig. 2(b)]. The offset angle of matched lattice planes is $\sim 12.7^{\circ}$, resulting in the formation of a zigzag grain boundary at the interface [Fig. 2(b)]. When the offset angle on either side of the interface becomes sufficiently large, leading to a serious mismatch, a mixed interlayer will form between the two welded $\mathrm{ZnO} \mathrm{NWs}$ as required to satisfy minimization of the interfacial energy (See Fig. S2 in the supplementary material).

It has previously been shown that femtosecond laser irradiation enables local crystalline nanowelding by nonequilibrium recrystallization rather than through epitaxial recrystallization ${ }^{19}$ and introduces point defects during rapid melting and solidification under high (a)
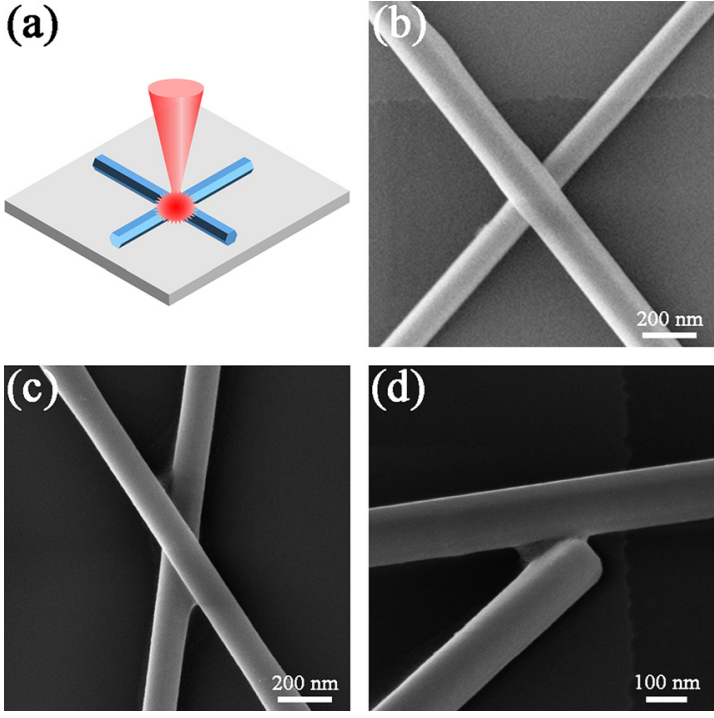

FIG. 1. (a) Schematic of the general experimental setup for optical nanowelding. ZnO NWs (blue) are deposited on the silicon chip (gray), and femtosecond laser beam (red) is focused on the junction area. (b) SEM image of overlapping $\mathrm{ZnO} N W$ s before welding. (c) and (d) SEM images of X- and Y-shaped ZnO NW junctions after nanowelding. The fluence is $77.6 \mathrm{~mJ} \mathrm{~cm}^{-2}$, and the irradiation time is $30 \mathrm{~s}$.

intensity irradiation. ${ }^{20}$ Figure $2(\mathrm{~d})$ shows that the concentration of point defects is enhanced at the interface, while there is no evidence for an increase in defect density away from the junction [Fig. 2(c)]. Further information on the properties of the point defects can be
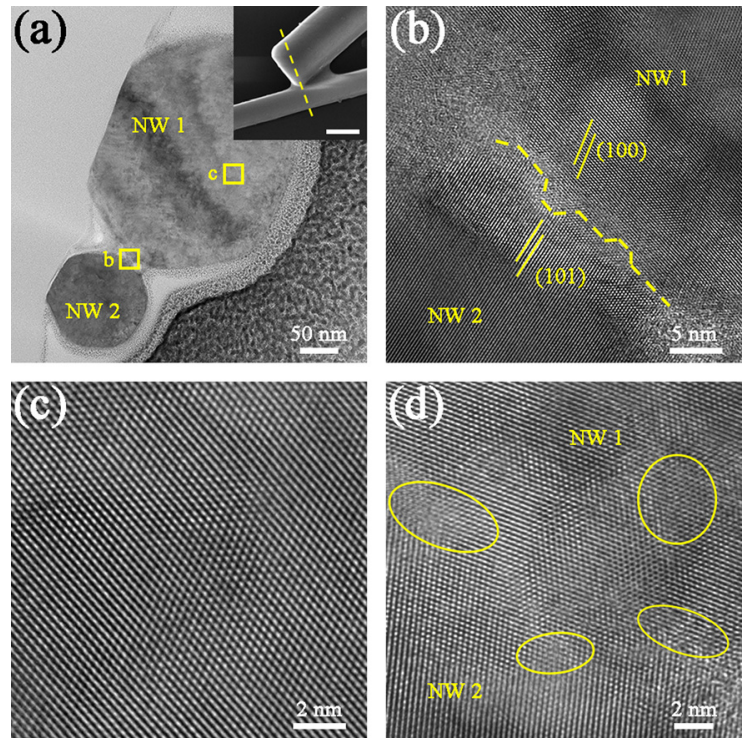

FIG. 2. (a) A TEM lamella image of a welding spot along the dashed line in the inset. The inset scale bar is $200 \mathrm{~nm}$. (b) and (c) show expanded images of the rectangular areas in (a). The dashed line in the inset of (b) shows the interface between two welded ZnO NWs. (d) shows the expanded image of the zigzag boundary area in (b). The solid circles indicate the location of point defects. 
obtained via ultraviolet (UV)-visible absorbance spectroscopy. Figure 3(a) shows that the band edge in the unirradiated $\mathrm{ZnO}$ NWs occurs at $375 \mathrm{~nm}$. The corresponding bandgap energy $\left(E_{\mathrm{g}}\right)$ can be calculated according to the following equation:

$$
(\alpha h \nu)^{2}=A\left(h \nu-E_{g}\right)^{n},
$$

where $\alpha$ is the optical absorption coefficient, $h \nu$ is the photon energy, $A$ is a constant, and $n$ is equal to 1 for a material with a direct bandgap. Linear extrapolation indicates that the bandgap energy is $3.29 \mathrm{eV}$ in unirradiated $\mathrm{ZnO}$ NWs [Fig. 3(b)]. Little change occurs in this plot after irradiation at low fluence $\left(<70 \mathrm{~mJ} \mathrm{~cm}^{-2}\right)$, but at higher fluence, tail states corresponding to an enhanced defect concentration extend the absorption edge to $\sim 600 \mathrm{~nm}$. The calculated bandgap energy becomes $3.26 \mathrm{eV}$ and $3.23 \mathrm{eV}$ after irradiation at fluences of $82.1 \mathrm{~mJ}$ $\mathrm{cm}^{-2}$ and $92.3 \mathrm{~mJ} \mathrm{~cm}{ }^{-2}$, respectively. This indicates that point defects, introducing energy levels within the bandgap, are generated during welding. As other studies suggest that these defects are most likely oxygen vacancies, ${ }^{22}$ annealing in air should remove these defects and reduce the visible absorption. Figure 3(a) shows that the visible absorption is reduced after annealing a previously irradiated sample $\left(92.3 \mathrm{~mJ} \mathrm{~cm}^{-2}\right)$ in air at $400^{\circ} \mathrm{C}$ for $2 \mathrm{~h}$, and the bandgap returns to $3.27 \mathrm{eV}$ [Fig. 3(b)], confirming that oxygen vacancies are introduced during intense femtosecond laser irradiation.

It should be noted that spectral changes are observed in an array of welded NWs, but the interfacial oxygen vacancies are present only in a subvolume of the samples near welded junctions. It can be seen that the cross section of $\mathrm{ZnO}$ NWs tends to be round at the top but remains hexagonal at the bottom [Fig. 2(a)]. This indicates that oxygen vacancies are also generated on the irradiated surface of the NWs, which can be associated with the observed enhancement in visible absorption. To gain more insight into oxygen vacancy distribution in
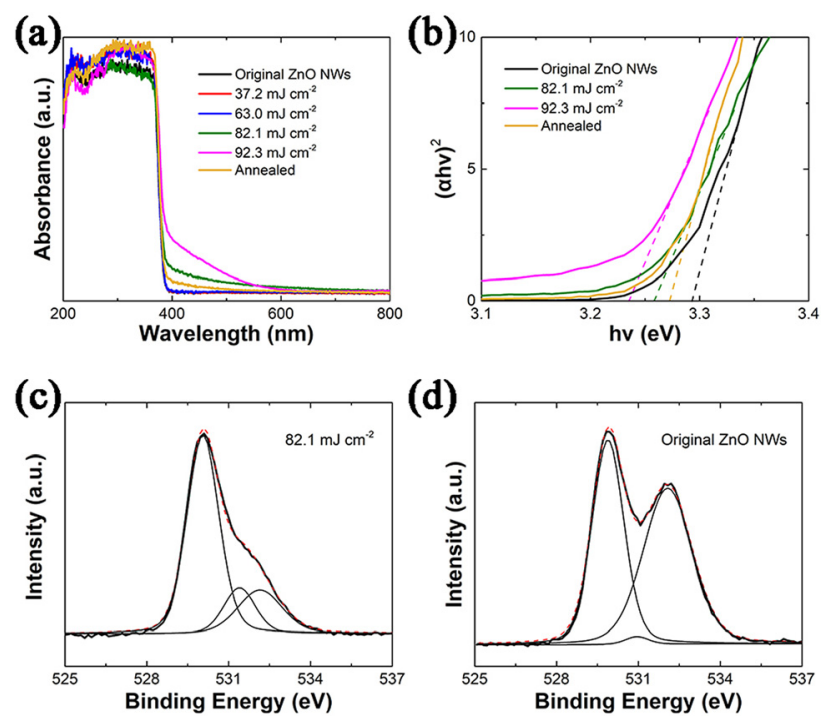

FIG. 3. (a) UV-visible absorbance spectra of ZnO NWs. (b) Calculated $(\alpha h \nu)^{2}$ vs $\mathrm{h} \nu$ curves of $\mathrm{ZnO} \mathrm{NWs}$. One of the irradiated samples (fluence $92.3 \mathrm{~mJ} \mathrm{~cm}^{-2}$ ) is then annealed in air at $400^{\circ} \mathrm{C}$ for $2 \mathrm{~h}$. 0 1s XPS high resolution spectra of (c) original $\mathrm{ZnO} N W s$ and (d) ZnO NWs after irradiation at a fluence of $82.1 \mathrm{~mJ} \mathrm{~cm} \mathrm{~cm}^{-2}$. welded samples, the chemical states of $\mathrm{O}$ have been investigated by micro X-ray photoelectron spectroscopy [Figs. 3(c) and 3(d)]. Components with binding energies of $530.1 \mathrm{eV}$ and $532.1 \mathrm{eV}$ are attributed to $\mathrm{O}^{2-}$ ions in the wurtzite structure of a hexagonal $\mathrm{Zn}^{2+}$ ion array ${ }^{23}$ and chemically absorbed oxygen on the NW surface, ${ }^{24}$ respectively. The peak centered at $531.4 \mathrm{eV}$ is associated with $\mathrm{O}^{2-}$ in oxygen deficient regions, which would be areas on the surface and at the welded junction with a high concentration of oxygen vacancies. ${ }^{24,25}$

The self-limited melting process in the welding of $\mathrm{ZnO} \mathrm{NWs}$ is similar to that in plasmonic welding of Ag NWs. ${ }^{1}$ However, as no surface/bulk plasmons can be excited in $\mathrm{ZnO}$ NWs under the current irradiation conditions, bonding must be due to some other selective excitation. To understand the nature of this excitation and the role it plays in localized nanowelding, photoluminescence spectra of $\mathrm{ZnO}$ NWs excited with $800 \mathrm{~nm}$ femtosecond laser pulses have been obtained [Fig. 4(a)]. The broadband emission peaking at $\sim 519 \mathrm{~nm}$ (peak C) can be attributed to a low concentration of intrinsic defects in the NWs. ${ }^{26}$ Peak B at $400 \mathrm{~nm}$ corresponds to the second harmonic of the incident femtosecond laser irradiation and occurs because of a nonlinear effect in $\mathrm{ZnO}$ NWs. ${ }^{27}$ The intense emission peak near the edge of the bandgap (Peak A, $389 \mathrm{~nm}$ ) can be attributed to the recombination of excitons through the exciton-exciton collision process. ${ }^{28}$ Figure 4(b) shows the intensity of peak A vs the excitation power on a log scale. The linear slope of 2.5 indicates that the excitons in $\mathrm{ZnO}$ are excited in a two-photon transition. ${ }^{27}$ It is noted that the excitation wavelength is $800 \mathrm{~nm}$ (corresponding to $1.55 \mathrm{eV}$ ), and the sum of energies of two photons is still less than the bandgap of $\mathrm{ZnO}(3.29 \mathrm{eV})$. However, under the extremely intense femtosecond laser excitation (the order of the peak power is $\sim 10^{2} \mathrm{GW} / \mathrm{cm}^{2}$ ), the intense light-mater interaction would result in strong Rabi oscillation, which could efficiently reduce the detuning between the bandgap energy and two-photon energy, thus enhancing the possibility of two-photon absorption. ${ }^{27}$

Two-photon absorption, during irradiation with intense femtosecond pulses, results in a high density of excitons in $\mathrm{ZnO}^{29}$ The density of these excitons, or mobile electron-hole pairs, increases rapidly under intense femtosecond excitation forming an electron-hole plasma. ${ }^{30}$ The energy in this plasma is deposited at lattice defect sites which tend to be concentrated on the surface of the NWs and in the contact area between two NWs. This can be seen in Figs. 1(c) and 1(d) which show that melting extends from the end as well as from the side of adjacent NWs. Energy deposition is then highly selective and nonuniform and occurs in regions of the NWs where the defect concentration is highest. This results in a localized decrease in the melting temperature. ${ }^{31}$ As the
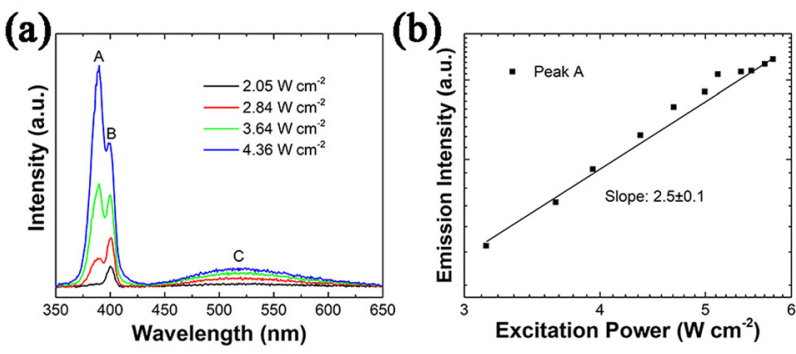

FIG. 4. (a) Photoluminescence emission spectra of $\mathrm{ZnO} N W s$ excited by intense femtosecond pulses at a wavelength of $800 \mathrm{~nm}$ as a function of incident fluence. (b) The intensity of peak A in (a) vs the excitation intensity. The solid line is the linear fit. 
system is exposed to many femtosecond laser pulses before melting occurs, it is likely that the defect concentration first builds up in the NWs near the eventual junction. This process occurs in localized regions where the optical field is enhanced. As the defect concentration increases, exciton trapping is facilitated at these locations, ${ }^{32}$ with attendant energy deposition leading to a localized rise in temperature. Generally, the nonthermal effect occurs during the ultrashort laser pulse-matter interaction. However, multipulse irradiation can result in accumulation of thermal energy and a significant temperature increase due to the thermalization of energy inside electron-hole plasma and relaxation of anharmonic excitation, such as melting and solidification. $^{33}$ In the present experiments, this self-limiting process corre-

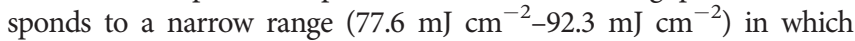
melting rather than ablation is dominant. Above this range, a slight increase in fluence leads to extensive ablation (see Fig. S3 in the supplementary material).

To study the photoelectrical properties of two welded $\mathrm{ZnO}$ NW junctions, Pt electrodes separated by $\sim 10 \mu \mathrm{m}$ were deposited by FIB as shown in the inset in Fig. 5(a), which ensures a stable conduction between the electrode and NW (see Fig. S4-S6 in the supplementary material). Before welding, the junction between the overlapping NWs is highly resistive $\left(\sim 10^{10} \Omega\right)$ in a dark environment [Fig. 5(a)]. After welding, the dark current shows a nonlinear I-V response with currents rising to $5.6 \mu \mathrm{A} /-4.1 \mu \mathrm{A}$ at a bias of $5 \mathrm{~V} /-5 \mathrm{~V}$. This change can be attributed to laser induced bonding between the two NWs. The initial deposited $\mathrm{ZnO}$ NWs are held together by a weak van der Waals force. In this case, a symmetric charge carrier depletion zone forms at the contacting surfaces due to the adsorption of oxygen on the NW surface. $^{34}$ A potential barrier, whose amplitude depends on the concentration of adsorbed oxygen, will limit the current density crossing the contact area. After welding, the formation of metallurgical (e.g., $\mathrm{Zn}-\mathrm{Zn})$ or covalent $(\mathrm{Zn}-\mathrm{O}$ and $\mathrm{O}-\mathrm{O})$ bonds in the contact area
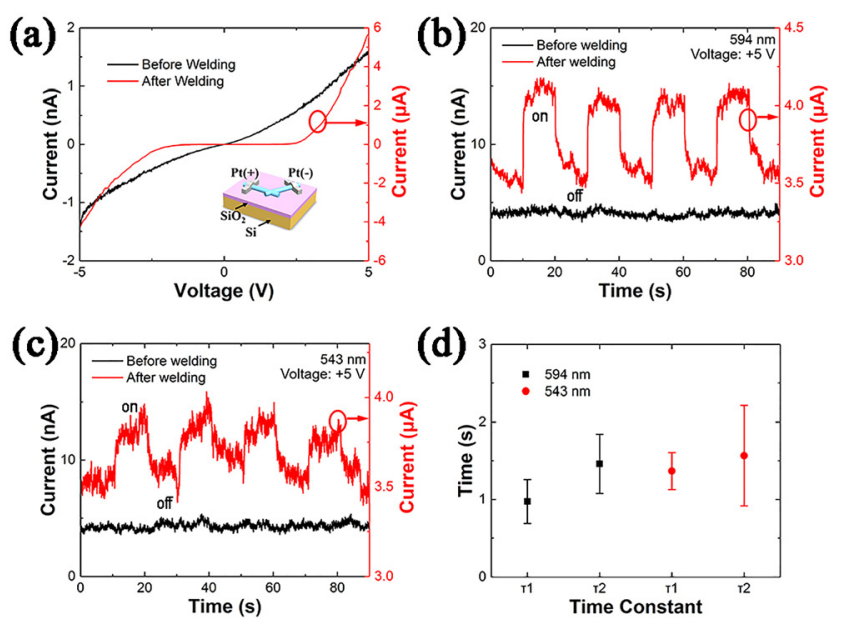

FIG. 5. (a) The I-V response of two $\mathrm{ZnO} N W$ junctions before and after femtosecond laser welding. The inset shows a schematic of the device (see the SEM image of the device in Fig. S7 in the supplementary material). The time-dependent current response of the $\mathrm{ZnO} N W$ junction under photoexcitation at (b) $594 \mathrm{~nm}$ and (c) $543 \mathrm{~nm}$. In each case, the LED power is $0.1 \mathrm{~mW}$ and the voltage is $+5 \mathrm{~V}$. (d) Time constants of response and recovery under visible illumination derived from fits to the emission data. eliminates adsorbed oxygen, producing a continuous structure. This lowers the barrier height and facilitates the transportation of charge carriers across the junction.

Figures 5(b) and 5(c) show the current response biased at $+5 \mathrm{~V}$ of a welded junction between two $\mathrm{ZnO}$ NWs excited with a number of light on/off cycles of visible light. Before welding, the device has no photocurrent response at either excitation wavelength. The subsequent photoresponse can then be attributed to the junction formed by femtosecond laser irradiation. The experimental data can be fit using the following exponential growth and decay curves:

$$
\begin{aligned}
& I(t)=I_{0}+A_{1}\left(1-e^{t / \tau_{1}}\right) \\
& I(t)=I_{0}+A_{2} e^{-t / \tau_{2}},
\end{aligned}
$$

where $I_{0}$ is the dark current, $A_{1}$ and $A_{2}$ are positive constants, and $\tau_{1}$ and $\tau_{2}$ are time constants for the rise and decay of the photogenerated currents, respectively. The fit parameters are given in Fig. 5(d) and are typically $\sim 1-1.5 \mathrm{~s}$. A reproducible photocurrent response is observed for multiple on/off cycles, indicating that the nanowelded junction is robust.

In summary, we have demonstrated a method for welding $\mathrm{ZnO}$ NWs, which utilizes two-photon absorption induced by femtosecond laser irradiation to create an electron-hole plasma via excitation of excitonic states. Trapping of electron-hole plasma energy at lattice defects causes localized heating and melting at the junction between the two NWs, resulting in the formation of a weld. During the nonequilibrium solidification process, oxygen vacancies are generated in the melt region, introducing defect levels in the $\mathrm{ZnO} \mathrm{NWs}$ that enhance optical absorption in the visible region (400-600 nm). Due to the decrease in the interfacial barrier, the junction formed by nanowelding between two $\mathrm{ZnO}$ NWs exhibits an improvement in dark conductivity of 4 orders of magnitude at a bias voltage of $+5 \mathrm{~V}$. The photogenerated currents also show fast responses and recovery under 543 and $594 \mathrm{~nm}$ excitation. It is expected that excitonic nanowelding of semiconductor materials with femtosecond laser irradiation has much promise for the assembly of large-area networks and in the development of functional nanoelectronic devices.

See the supplementary material for all the experimental details and other supporting figures.

This work was supported by the National Key Research and Development Program of China (No. 2017YFB1104900) and National Natural Science Foundation of China (Grant Nos. 51520105007 and 51775299). The author Songling Xing thanks the State Key Laboratory of Tribology in Tsinghua University for making TEM lamella and Professor Peng Peng and Ph.D. student Ming Xiao for fruitful discussion.

\section{REFERENCES}

${ }^{7}$ S. Han, S. Hong, J. Ham, J. Yeo, J. Lee, B. Kang, P. Lee, J. Kwon, S. S. Lee, M. Yang, and S. H. Ko, Adv. Mater. 26, 5808-5814 (2014).

${ }^{2}$ S. Hong, H. Lee, J. Lee, J. Kwon, S. Han, Y. D. Suh, H. Cho, J. Shin, J. Yeo, and S. H. Ko, Adv. Mater. 27, 4744-4751 (2015).

${ }^{3}$ L. Lin, L. Liu, K. Musselman, G. Zou, W. W. Duley, and Y. N. Zhou, Adv. Funct. Mater. 26, 5979-5986 (2016).

${ }^{4}$ E. C. Garnett, W. Cai, J. J. Cha, F. Mahmood, S. T. Connor, M. G. Christoforo, Y. Cui, M. D. McGehee, and M. L. Brongersma, Nat. Mater. 11, 241 (2012). 
${ }^{5}$ K. Keramatnejad, Y. Zhou, D. Li, H. R. Golgir, X. Huang, Q. Zhou, J. Song, S. Ducharme, and Y. Lu, Adv. Mater. Interfaces 4, 1700294 (2017).

${ }^{6}$ K. Qu, H. Zhang, Q. Lan, X. Deng, X. Ma, Y. Huang, J. Zhang, H. Ma, B. J. Inkson, D. Xue, and Y. Peng, J. Mater. Chem. C 3, 11311-11317 (2015).

${ }^{7}$ L. Zhang, Y. Tang, Q. Peng, T. Yang, Q. Liu, Y. Wang, Y. Li, C. Du, Y. Sun, L. Cui, F. Yang, T. Shen, Z. Shan, and J. Huang, Nat. Commun. 9, 96 (2018).

${ }^{8}$ A. Bo, J. Alarco, H. Zhu, E. R. Waclawik, H. Zhan, and Y. Gu, ACS Appl. Mater. Interfaces 9, 9143-9151 (2017).

${ }^{9}$ Y. Son, J. Yeo, H. Moon, T. Lim, S. Hong, K. H. Nam, S. Yoo, C. P. Grigoropoulos, D. Yang, and S. H. Ko, Adv. Mater. 23, 3176-3181 (2011).

${ }^{10}$ S. Xing, L. Lin, G. Zou, L. Liu, P. Peng, A. Wu, W. W. Duley, and Y. Norman Zhou, Nanotechnology 28, 405302 (2017).

${ }^{17}$ G. Liu, Q. Li, and M. Qiu, J. Phys.: Conf. Ser. 680, 012027 (2016).

${ }^{12}$ S. H. Ko, I. Park, H. Pan, N. Misra, M. S. Rogers, C. P. Grigoropoulos, and A. P. Pisano, Appl. Phys. Lett. 92, 154102 (2008).

${ }^{13}$ J. Kwon, S. Hong, H. Lee, J. Yeo, S. S. Lee, and S. H. Ko, Nanoscale Res. Lett. 8, 489 (2013).

${ }^{14}$ S. H. Ko, D. Lee, H. W. Kang, K. H. Nam, J. Y. Yeo, S. J. Hong, C. P. Grigoropoulos, and H. J. Sung, Nano Lett. 11, 666-671 (2011).

${ }^{15}{ }^{15}$. Özgür, Y. I. Alivov, C. Liu, A. Teke, M. A. Reshchikov, S. Doğan, V. Avrutin, S. J. Cho, and H. Morkoç, J. Appl. Phys. 98, 041301 (2005).

${ }^{16} \mathrm{C}$. Hsu, Y. Lin, L. Wang, T. Hsueh, S. Chang, and S. Chang, ACS Appl. Mater. Interfaces 9, 14935-14944 (2017).

${ }^{17}$ A. Šutka, T. Käämbre, R. Pärna, I. Juhnevica, M. Maiorov, U. Joost, and V. Kisand, Solid State Sci. 56, 54-62 (2016).

${ }^{18}$ Q. Guo, Q. Zhang, H. Wang, and Z. Zhao, Catal. Commun. 103, 24-28 (2018).
${ }^{19}$ Q. Nian, M. Saei, Y. Xu, G. Sabyasachi, B. Deng, Y. P. Chen, and G. J. Cheng, ACS Nano 9, 10018-10031 (2015).

${ }^{20}$ V. I. Emel'yanov and D. V. Babak, Appl. Phys. A 74, 797-805 (2002).

${ }^{21}$ P. Ghamgosar, F. Rigoni, S. You, I. Dobryden, M. G. Kohan, A. L. Pellegrino, I. Concina, N. Almqvist, G. Malandrino, and A. Vomiero, Nano Energy 51, 308-316 (2018).

${ }^{22}$ A. Janotti and C. G. Van de Walle, Phys. Rev. B 76, 165202 (2007).

${ }^{23}$ S. Park, T. Ikegami, and K. Ebihara, Thin Solid Films 513, 90-94 (2006).

${ }^{24}$ H. Fan, S. Yang, P. Zhang, H. Wei, X. Liu, C. Jiao, Q. Zhu, Y. Chen, and Z. Wang, Chin. Phys. Lett. 24, 2108-2111 (2007).

${ }^{25}$ G. R. Dillip, A. N. Banerjee, V. C. Anitha, B. Raju, S. W. Joo, and B. K. Min, ACS Appl. Mater. Interfaces 8, 5025-5039 (2016).

${ }^{26} \mathrm{M}$. H. Huang, Y. Wu, H. Feick, N. Tran, E. Weber, and P. Yang, Adv. Mater. 13, 113-116 (2001).

${ }^{27}$ C. F. Zhang, Z. W. Dong, G. J. You, R. Y. Zhu, S. X. Qian, H. Deng, H. Cheng, and J. C. Wang, Appl. Phys. Lett. 89, 042117 (2006).

${ }^{28}$ D. M. Bagnall, Y. F. Chen, Z. Zhu, T. Yao, S. Koyama, M. Y. Shen, and T. Goto, Appl. Phys. Lett. 70, 2230-2232 (1997).

${ }^{29}$ E. Hendry, M. Koeberg, and M. Bonn, Phys. Rev. B 76, 045214 (2007).

${ }^{30}$ B. P. Mehl, J. R. Kirschbrown, R. L. House, and J. M. Papanikolas, J. Phys. Chem. Lett. 2, 1777-1781 (2011).

${ }^{31}$ K. M. Wong, S. M. Alay-e-Abbas, Y. Fang, A. Shaukat, and Y. Lei, J. Appl. Phys. 114, 034901 (2013).

${ }^{32}$ W. Zhou and H. Fu, Inorg. Chem. Front. 5, 1240-1254 (2018).

${ }^{33}$ V. E. Grudzev and A. S. Gruzdeva, Proc. SPIE 4065, 62-73 (2000).

${ }^{34}$ W. J. Park, K. J. Choi, M. H. Kim, B. H. Koo, J. Lee, and J. M. Baik, ACS Appl. Mater. Interfaces 5, 6802-6807 (2013). 